\title{
ON HARDY-LITTLEWOOD SERIES
}

\author{
BY \\ MARY WEISS( $\left.{ }^{1}\right)$
}

1. In this paper we will discuss the behavior of the series

$$
\begin{aligned}
& \sum_{1}^{\infty} n^{-1 / 2} \exp (i \beta n \log n+i n \theta), \\
& \sum_{1}^{\infty} n^{-1 / 2}(\log n)^{-1 / 2} \exp (i \beta n \log n+i n \theta)
\end{aligned}
$$

which were first considered by Hardy and Littlewood [1]. Here $\beta$ is real and nonzero; without loss of generality we may assume $\beta>0$. Hardy and Littlewood have proved that the Abel means of (1.1) are unbounded almost everywhere. They have also given a functional relation connecting the series with certain lacunary power series. Using the Law of the Iterated Logarithm and the Central Limit Theorem for lacunary series, both of which will be stated later, we obtain sharp estimates for the behavior of the partial sums and Abel means of (1.1) and (1.2).

The series (1.1) and (1.2) have also been investigated by Paley [1], who gave a different proof of the functional equation of Hardy and Littlewood. In this paper it is somewhat more convenient to use Paley's results.

The main results of this paper are the following two theorems.

ThEOREM 1. If

$$
\begin{aligned}
& S_{N}(\theta)=\sum_{n=1}^{N} n^{-1 / 2} \exp (i \beta n \log n+i n \theta), \\
& S_{\rho}(\theta)=\sum_{n=1}^{\infty} n^{-1 / 2} \rho^{n} \exp (i \beta n \log n+i n \theta), \\
& S_{N}^{*}(\theta)=\sum_{n=1}^{N} n^{-1 / 2}(\log n)^{-1 / 2} \exp (i \beta n \log n+i n \theta), \\
& S_{\rho}^{*}(\theta)=\sum_{n=1}^{\infty} n^{-1 / 2}(\log n)^{-1 / 2} \rho^{n} \exp (i \beta n \log n+i n \theta),
\end{aligned}
$$

then almost everywhere in $\theta$ we have the following relations:

Presented to the Society, February 25, 1956, under the title On the Littlewood series $\sum n^{-1 / 2} \exp (i \beta n+i n \theta)$; received by the editors August $19,1957$.

(1) The research resulting in this paper was supported by the Office of the Scientific Research of the Air Force under contract AF18(600)-1111. 


$$
\begin{aligned}
& \limsup _{N \rightarrow \infty} \frac{\left|S_{N}(\theta)\right|}{(\log N \cdot \log \log \log N)^{1 / 2}}=1, \\
& \limsup _{\rho \rightarrow 1} \frac{\left|S_{\rho}(\theta)\right|}{\left(\log \frac{1}{(1-\rho)} \cdot \log \log \log \frac{1}{(1-\rho)}\right)^{1 / 2}}=1 \text {, }
\end{aligned}
$$

$$
\limsup _{N \rightarrow \infty} \frac{\left|S_{N}^{*}(\theta)\right|}{(\log \log N \cdot \log \log \log \log N)^{1 / 2}}=1,
$$

$$
\limsup _{\rho \rightarrow 1} \frac{\left|S_{\rho}^{*}(\theta)\right|}{\left(\log \log \frac{1}{(1-\rho)} \cdot \log \log \log \log \frac{1}{(1-\rho)}\right)^{1 / 2}}=1 .
$$

Theorem 2. Let $E$ be a set of positive measure contained in $(0,2 \pi)$. If $S_{N}(\theta), S_{\rho}(\theta), S_{N}^{*}(\theta)$ and $S_{\rho}^{*}(\theta)$ have the same meaning as in Theorem 1 , and if $F_{N}(\xi, \eta), F_{\rho}(\xi, \eta), F_{N}^{*}(\xi, \eta)$ and $F_{\rho}^{*}(\xi, \eta)$ are the two dimensional distribution functions of

$$
\begin{aligned}
\frac{S_{N}(\theta)}{\left(2^{-1} \log N\right)^{1 / 2}}, & \frac{S_{\rho}(\theta)}{\left(2^{-1} \log 1 /(1-\rho)\right)^{1 / 2}}, \frac{S_{N}^{*}(\theta)}{\left(2^{-1} \log \log N\right)^{1 / 2}}, \\
& \frac{S_{\rho}^{*}(\theta)}{\left(2^{-1} \log \log 1 /(1-\rho)\right)^{1 / 2}}
\end{aligned}
$$

over the set $E$, that is, for example

$$
F_{N}(\xi, \eta)=\left|\left\{\theta: \theta \in E ; \propto \frac{S_{N}(\theta)}{\left(2^{-1} \log N\right)^{1 / 2}}<\xi ; \mathfrak{g} \frac{S_{N}(\theta)}{\left(2^{-1} \log N\right)^{1 / 2}}<\eta\right\}\right|
$$

then

$$
\begin{gathered}
F_{N}(\xi, \eta) /|E| \underset{N \rightarrow \infty}{\longrightarrow} G(\xi, \eta), \\
F_{\rho}(\xi, \eta) /|E| \underset{\rho \rightarrow 1}{\longrightarrow} G(\xi, \eta), \\
F_{N}^{*}(\xi, \eta) /|E| \underset{N \rightarrow \infty}{\longrightarrow} G(\xi, \eta), \\
F_{\rho}^{*}(\xi, \eta) /|E| \underset{\rho \rightarrow 1}{\longrightarrow} G(\xi, \eta),
\end{gathered}
$$

where $G(\xi, \eta)$ is the two dimensional Gaussian distribution.

2. In the proof of the above two theorems we will use the following theorem of Paley [1]:

Theorem A. If 


$$
\begin{aligned}
a & =\exp \left(2 \pi \beta^{-1}\right) ; \quad z=\rho e^{i \theta} ; \quad z^{\prime}=\rho^{\prime} e^{i \theta^{\prime}}, \\
\rho^{\prime} & =\rho \exp \left(-1-\theta \beta^{-1}\right) ; \quad \theta^{\prime}=-\beta \exp \left(-1-\theta \beta^{-1}\right)
\end{aligned}
$$

then

$$
\begin{aligned}
\sum_{n=1}^{\infty} n^{-1 / 2}\left(\log n+1+\theta \beta^{-1}\right)^{\lambda} \exp (i \beta n & \log n) z^{n} \\
& =e^{\pi i / 4}\left(2 \pi \beta^{-1}\right)^{\lambda+1 / 2} \sum_{\nu=1}^{\infty} \nu^{\lambda}\left(z^{\prime}\right)^{a^{\nu}}+R
\end{aligned}
$$

where $R$ is a function continuous in the circle $\rho \leqq 1$. Also, if $\rho=1$,

$$
\begin{aligned}
\sum_{n=1}^{N} n^{-1 / 2}(\log n+ & \left.1+\theta \beta^{-1}\right)^{\lambda} \exp (i \beta n \log n+i n \theta) \\
& =e^{\pi i / 4}\left(2 \pi \beta^{-1}\right)^{\lambda+1 / 2} \sum_{\nu=1}^{\beta(2 \pi)^{-1} \log N} \nu^{\lambda}\left(z^{\prime}\right)^{a^{\nu}}+O\left(\mu^{\lambda}\right)+O(1)
\end{aligned}
$$

where $\mu$ denotes the integer next below $\beta(2 \pi)^{-1} \log N$.

We also need the theorem of the Law of the Iterated Logarithm (see M. Weiss [1]).

Theorem B. If

$$
S(x)=\sum_{k=1}^{\infty} c_{k} e^{i n_{k} x}
$$

is a lacunary power series, that is $n_{k+1} / n_{k}>q>1$, with coefficients satisfying

$$
\max _{1 \leqq K \leqq N}\left|c_{k}\right|=o\left\{\frac{B_{N}}{\left(\log \log B_{N}\right)^{1 / 2}}\right\}
$$

where

$$
B_{N}=\left\{\sum_{k=1}^{N}\left|c_{k}\right|^{2}\right\}^{1 / 2} \rightarrow \infty
$$

then

$$
\lim \sup \frac{S_{N}(x)}{\left(B_{N}^{2} \log \log B_{N}\right)^{1 / 2}}=1
$$

almost everywhere. Furthermore, if

$$
S_{r}(x)=\sum_{k=1}^{\infty} c_{k} e^{i n_{k} x} r^{n_{k}}, \quad B_{\boldsymbol{r}}=\left(\sum_{k=1}^{\infty} c_{k}^{2} r^{2 n_{k}}\right)^{1 / 2}
$$


then

$$
\lim \sup \frac{S_{r}(x)}{\left(B_{r}^{2} \log \log B_{r}\right)^{1 / 2}}=1
$$

amost everywhere.

3. In this section we will prove (1.7) and (1.8) of Theorem 1.

If we take $\lambda=0$, Equation (2.1) becomes

$$
\sum_{n=1}^{N} n^{-1 / 2} \exp (i \beta n \log n+i n \theta)=e^{\pi i / 4}\left(2 \pi \beta^{-1}\right)^{1 / 2} \sum_{\nu=1}^{\beta(2 \pi)^{-1} \log N} e^{i \theta^{\prime} a^{\nu}}+O(1) .
$$

The left side of the above equation is (1.3) while the right side is the $N$ th partial sum of the lacunary power series

$$
\sum_{\nu=1}^{\infty} e^{i \theta^{\prime} a^{\nu}}
$$

plus a bounded term, where $N^{\prime}=\beta(2 \pi)^{-1} \log N$. We see that $B_{N^{\prime}}^{2}$ $=\beta(2 \pi)^{-1} \log N$, and hence by Theorem B

$$
\lim \sup \frac{\left|\sum_{\nu=1}^{\beta(2 \pi)^{-1} \log N} e^{i \theta^{\prime} a^{\nu}}\right|}{\left(\beta(2 \pi)^{-1} \log N \cdot \log \log \log N\right)^{1 / 2}}=1
$$

almost everywhere in $\theta^{\prime}$, and hence by (3.1),

$$
\lim \sup \frac{\left|S_{N}(\theta)\right|}{(\log N \cdot \log \log \log N)^{1 / 2}}=1
$$

almost everywhere in $\theta$.

We now turn to the proof of (1.8). For $\lambda=0, \rho$ fixed, the left side of (2.1) is simply $S_{\rho}(\theta)$. However, the right side of (2.1) is not an Abel mean of the series (3.2), because when $\rho$ is constant $\rho^{\prime}$ is not. Therefore, in order to apply Theorem B to the right side of (2.1) we must replace $\rho^{\prime}$ by $\rho$. That is, we write

$$
\begin{aligned}
S_{\rho}(\theta)= & e^{\pi i / 4}\left(2 \pi \beta^{-1}\right)^{1 / 2} \sum_{\nu=1}^{\infty} e^{i \theta^{\prime} a^{\nu} \rho^{a^{\nu}}} \\
& \left.+e^{\pi i / 4}\left(2 \pi \beta^{-1}\right)^{1 / 2} \sum_{\nu=1}^{\infty} e^{i \theta^{\prime} a^{\nu}\left(\rho^{\prime} a^{\nu}\right.}-\rho^{a^{\nu}}\right)+R .
\end{aligned}
$$

Now we estimate the "error" term

$$
P=\left|\sum_{\nu=1}^{\infty} e^{i \theta^{\prime} a^{\nu}}\left(\rho^{a^{\nu}}-\rho^{a^{\nu}}\right)\right|
$$

Letting $\rho^{\prime}=\rho+\delta$, where $\delta$ is of course a function of $\rho$ and $\theta$, we obtain 


$$
\begin{aligned}
P & \leqq \delta \sum_{\nu=1}^{\infty} a^{\nu}(\rho+\delta)^{a^{\nu}-1} \leqq A \delta /(1-(\rho+\delta)) \\
& \leqq A \frac{\left(\rho^{\alpha}-\rho\right)}{1-\rho^{\alpha}}
\end{aligned}
$$

where $\alpha=\exp \left(-1-\theta \beta^{-1}\right)$. Since $\alpha$ is positive and bounded away from zero, it follows that

$$
\lim \frac{\rho^{\alpha}-\rho}{1-\rho^{\alpha}}=\frac{1-\alpha}{\alpha} .
$$

Thus $P$ is a bounded function of $\rho, \theta$. Applying Theorem $\mathrm{B}$ to the series $\sum_{\nu=1}^{\infty} e^{i \theta^{\prime} a^{\nu}}$ for which

we obtain

$$
B_{\rho}^{2}=\sum_{1}^{\infty} \rho^{2 a^{\nu}} \sim \frac{1}{\log a} \log \frac{1}{1-\rho}=\beta(2 \pi)^{-1} \log \frac{1}{1-\rho},
$$

$$
\lim \sup \frac{\left|\sum_{\nu=1}^{\infty} e^{i \theta^{\prime} a^{\nu} \rho^{a^{\nu}}}\right|}{\left((2 \pi)^{-1} \log 1 /(1-\rho) \log \log \log 1 /(1-\rho)\right)^{1 / 2}}=1
$$

almost everywhere in $\theta^{\prime}$.

Equations (3.3) and (3.5), together with the boundedness of $P$ give us

$$
\lim \sup \frac{\left|S_{\rho}(\theta)\right|}{(\log 1 /(1-\rho) \log \log \log 1 /(1-\rho))^{1 / 2}}=1
$$

almost everywhere in $\theta$.

4. In this section we consider equations (1.9) and (1.10). Using (2.2) for $\lambda=-1 / 2$ and noting that

$$
B_{N^{\prime}}^{* 2}=\sum_{\nu=1}^{\beta(2 \pi)^{-1} \log N} \nu^{-1} \sim \log \log N
$$

Where $N^{\prime}=\beta(2 \pi)^{-1} \log N$, we obtain, in exactly the same manner as for (1.7),

(4.2) $\lim \sup \frac{\left|\sum_{n=1}^{N} n^{-1 / 2}\left(\log n+1+\theta \beta^{-1}\right)^{1 / 2} \exp (i \beta n \log n+i n \theta)\right|}{(\log \log N \cdot \log \log \log \log N)^{1 / 2}}=1$.

Using (2.1) for $\lambda=-1 / 2$ and noting that

$$
B_{\rho}^{* 2}=\sum_{\nu=1} \nu^{-1} \rho^{2 a^{\nu}} \sim \log \log \left(\frac{1}{1-\rho}\right)
$$

we obtain 
(4.4) $\lim \sup \frac{\left|\sum_{n=1}^{N} n^{-1 / 2}\left(\log n+1+\theta \beta^{-1}\right)^{-1 / 2} \exp (i \beta n \log n) z^{n}\right|}{\left((\log \log (1 /(1-\rho)) \log \log \log \log (1 /(1-\rho)))^{1 / 2}\right.}=1$

almost everywhere in $\theta$.

Now in order to obtain (1.9) and (1.10) from (4.2) and (4.4) we substitute $(\log n)^{-1 / 2}$ for $\left(\log n+1+\theta \beta^{-1}\right)^{-1 / 2}$ and justify that the remainder is small. We will use the following theorem of Hardy and Littlewood (see Zygmund [1]).

Theorem. The Nth partial sum of the series

$$
\sum_{n=1}^{\infty} \exp (i \beta n \log n+i n \theta)
$$

is $O\left(N^{1 / 2}\right)$ uniformly in $\theta$.

We write

$$
\sum_{n=1}^{N} n^{-1 / 2}(\log n)^{-1 / 2} \exp (i \beta n \log n+i n \theta)
$$

$(4.5)=\sum_{n=1}^{N} n^{-1 / 2}\left(\log n+1+\theta \beta^{-1}\right)^{-1 / 2} \exp (i \beta n \log n+i n \theta)$

$+\sum_{n=1}^{N} n^{-1 / 2}\left((\log n)^{-1 / 2}-\left(\log n+1+\theta \beta^{-1}\right)^{-1 / 2}\right) \exp (i \beta n \log n+i n \theta)$.

Applying Abel's transformation to the second sum on the right in the above equation we obtain,

$$
\begin{aligned}
\mid \sum_{n=1}^{N-1}\left\{n ^ { - 1 / 2 } \left[(\log n)^{-1 / 2}-\right.\right. & \left.(\log n+\alpha)^{-1 / 2}\right] \\
\left.-(n+1)^{-1 / 2}\left[(\log (n+1))^{-1 / 2}-(\log (n+1)+\alpha)^{-1 / 2}\right]\right\} \widetilde{S}_{n}(\theta) & \mid \\
& +\left|\widetilde{S}_{N}(\theta) N^{-1 / 2}\left[(\log N)^{-1 / 2}-(\log N+\alpha)^{-1 / 2}\right]\right|
\end{aligned}
$$

where $\alpha=1+\theta \beta^{-1}$ and $\widetilde{S}_{n}(\theta)=\sum_{k=1}^{n} \exp (i \beta k \log k+i k \theta)$. For some value $0 \leqq \gamma \leqq 1,(4.6)$ is equal to

$$
\left|\frac{1}{2} \sum_{n=1}^{N-1}(n+\gamma)^{-3 / 2}\left[(\log (n+\gamma))^{-3 / 2}-(\log (n+\gamma)+\alpha)^{-3 / 2}\right] \widetilde{S}_{n}(\theta)\right|
$$

$$
\begin{aligned}
& +\mid \frac{1}{2} \sum_{n=1}^{N-1}(n+\gamma)^{-3 / 2}\left[\left(\log (n+\gamma)^{-1 / 2}-(\log (n+\gamma)+\alpha)^{-1 / 2}\right] \widetilde{S}_{n}(\theta) \mid\right. \\
& +\left|N^{-1 / 2}\left[(\log N)^{-1 / 2}-(\log N+\alpha)^{-1 / 2}\right] \widetilde{S}_{N}(\theta)\right| .
\end{aligned}
$$


Upon replacing $S_{n}(\theta)$ by $O\left(n^{1 / 2}\right)$ we see that (4.7) is less than

$C \sum_{n=1}^{N-1} n^{-1}\left[(\log n)^{-3 / 2}+\left|(\log (n+\gamma))^{-1 / 2}-(\log (n+\gamma)+\alpha)^{-1 / 2}\right|\right]$.

$$
\leqq C_{1} \sum_{n=1}^{N-1} n^{-1}(\log n)^{-3 / 2} \text {. }
$$

Since $\sum_{n=1}^{\infty} n^{-1}(\log n)^{-3 / 2}<\infty$, we have

$$
S_{N}^{*}(\theta)=\sum_{n=1}^{N} n^{-1 / 2}\left(\log n+1+\beta^{-1} \theta\right)^{-1 / 2} \exp (i \beta n \log n+i n \theta)+O(1)
$$

and from Equations (4.2) and (4.4) we obtain (1.9) and (1.10).

5 . We now turn to the proof of Theorem 2. We will use the following theorem of Salem and Zygmund ([1] and [2]).

Theorem C. If

$$
\sum_{k=1}^{\infty} c_{k} e^{i n_{k} \theta}
$$

is a lacunary power series satisfying

$$
C_{N}=\left(\frac{1}{2} \sum_{k=1}^{N}\left|c_{k}\right|^{2}\right)^{1 / 2} ; \frac{c_{N}}{C_{N}} \rightarrow 0
$$

and $E$ is a set of positive measure contained in $(0,2 \pi)$, then

$$
\frac{F_{N}(\xi, \eta)}{|E|} \rightarrow G(\xi, \eta) ; \quad \frac{F_{\rho}(\xi, \eta)}{|E|} \rightarrow G(\xi, \eta)
$$

where $F_{N}(\xi, \eta)$ and $F_{\rho}(\xi, \eta)$ are the two dimensional distribution functions respectively of

$$
\frac{\sum_{k=1}^{N} c_{k} e^{i n_{k}}}{C_{N}} \text { and } \frac{\sum_{k=1}^{\infty} c_{k} \rho^{n k} e^{i n_{k} \theta}}{C_{\rho}}
$$

and $G(\xi, \eta)$ is the two dimensional Gaussian distribution.

We note that it is sufficient to prove Theorem 2 in the case where $E$ is an interval. The proof will be an immediate consequence of (2.1) and the following lemma.

Lemma. Let $P_{N}(\theta)$ be a sequence of functions defined on the unit circle and let $Q_{N}\left(\theta^{\prime}\right)=P_{N}(\theta)$ where

$$
\theta^{\prime}=g(\theta)
$$

is a one-one mapping of the class $C^{\prime}$. 
Suppose furthermore, that for any interval $E^{\prime}$ on the $\theta^{\prime}$ circle, the two dimensional distribution function of $Q_{N}\left(\theta^{\prime}\right)$, where $\theta^{\prime}$ is restricted to $E^{\prime}$, converges to the Gaussian. Then if $E$ is the image of $E^{\prime}$, under $g^{-1}\left(\theta^{\prime}\right)=f\left(\theta^{\prime}\right)$, the two dimensional distribution function of $P_{N}(\theta)$, where $\theta$ is restricted to $E$, converges to the Gaussian.

If $U^{\prime}$ is any measurable subset of the $\theta^{\prime}$ circle and $U$ its image in the $\theta$ circle then

$$
|U|=\int_{U^{\prime}} f^{\prime}\left(\theta^{\prime}\right) d \theta^{\prime}
$$

Hence if we divide the interval $E^{\prime}$ into $k$ subintervals $E_{i}^{\prime}$ so small that $f^{\prime}\left(\theta^{\prime}\right)$ varies by less than $\delta$ over each $E_{i}^{\prime}$, we will have for any measurable subset $U_{i}^{\prime}$ of $E_{i}^{\prime}$,

$$
\left|U_{i}\right|(1-\delta) \leqq f^{\prime}\left(\theta_{i}^{\prime}\right)\left|U_{i}^{\prime}\right| \leqq\left|U_{i}\right|(1+\delta),
$$

where $\theta_{i}^{\prime}$ is the left hand end point of $E_{i}^{\prime}$ and $E_{i}$ and $U_{i}$ are, of course, the images of $E_{i}^{\prime}$ and $U_{i}^{\prime}$. We write

$$
E_{i, N}^{\prime}(\xi, \eta)=\left\{\theta^{\prime}: \theta^{\prime} \in E_{i}^{\prime}, \Re\left(Q_{N}\left(\theta^{\prime}\right)\right)<\xi, \mathscr{I}\left(Q_{N}\left(\theta^{\prime}\right)\right)<\eta\right\}
$$

and note that if $E_{i, N}$ is the image of $E_{i, N}^{\prime}$, then

$$
E_{i, N}(\xi, \eta)=\left\{\theta: \theta \in E_{i}, R\left(P_{N}(\theta)\right)<\xi, \mathscr{g}\left(P_{N}(\theta)\right)<\eta\right\} .
$$

We write

$$
E_{N}(\xi, \eta)=\left\{\theta: \theta \in E, \Re\left(P_{N}(\theta)\right)<\xi, \mathscr{g}\left(P_{N}(\theta)\right)<\eta\right\}
$$

and note that

$$
\left|E_{N}(\xi, \eta)\right|=\sum_{i=1}^{k}\left|E_{i, N}(\xi, \eta)\right| .
$$

Now the conclusion of the lemma is simply that

$$
\frac{\left|E_{N}(\xi, \eta)\right|}{|E|} \rightarrow G(\xi, \eta) \text {. }
$$

From (5.2) it follows that

$$
\frac{\left|E_{i, N}\right|}{\left|E_{i}\right|}(1-\epsilon) \leqq \frac{\left|E_{i, N}^{\prime}\right|}{\left|E_{i}^{\prime}\right|} \leqq \frac{\left|E_{i, N}\right|}{\left|E_{i}\right|}(1+\epsilon)
$$

where $\epsilon$ is such that $((1+\delta) /(1-\delta))(1-\epsilon) \leqq 1 ;((1-\delta) /(1+\delta))(1+\epsilon) \geqq 1$. Hence, using 


$$
\frac{\left|E_{N}\right|}{|E|}=\frac{\sum_{i=1}^{k}\left|E_{i, N}\right|}{|E|}=\sum_{i=1}^{k} \frac{\left|E_{i, N}\right|}{\left|E_{i}\right|} \frac{\left|E_{i}\right|}{|E|},
$$

we have

$$
(1-\epsilon) \frac{\left|E_{N}\right|}{|E|} \leqq \sum_{i=1}^{k} \frac{\left|E_{i, N}^{\prime}\right|}{\left|E_{i}^{\prime}\right|} \frac{\left|E_{i}\right|}{|E|}=(1+\epsilon) \frac{\left|E_{N}\right|}{|E|} .
$$

By the hypotheses of the lemma,

$$
\lim _{N \rightarrow \infty} \sum_{i=1}^{k} \frac{\left|E_{i, N}^{\prime}(\xi, \eta)\right|}{\left|E_{i}^{\prime}\right|} \frac{\left|E_{i}\right|}{|E|}=G(\xi, \eta) \sum \frac{\left|E_{i}\right|}{|E|}=G(\xi, \eta) .
$$

Since $\epsilon$ can be made as small as we please, (5.5) together with (5.6) gives us (5.3).

Now we apply our lemma to the equation

$$
\begin{aligned}
P_{N}(\theta) & =\sum_{n=1}^{N} \frac{n^{-1 / 2} \exp (i \beta n \log n+i n \theta)}{\left(2^{-1} \log N\right)^{1 / 2}} \\
& =\frac{e^{\pi i / 4}\left(2 \pi \beta^{-1}\right)^{1 / 2} \sum_{\nu=1}^{\beta(2 \pi)^{-1} \log N} e^{i \theta^{\prime} a^{\nu}}}{\left(2^{-1} \log N\right)^{1 / 2}}+o(1) \\
& =Q_{N}\left(\theta^{\prime}\right)
\end{aligned}
$$

which comes directly from (2.2). Since by the theorem of Salem and Zygmund quoted above, $1 /\left|E^{\prime}\right|$ times the distribution function of

$$
\frac{e^{\pi i / 4}\left(2 \pi \beta^{-1}\right)^{1 / 2} \sum_{\nu=1}^{\beta(2 \pi)^{-1} \log N} e^{i \theta^{\prime} a^{\nu}}}{\left(2^{-1} \log N\right)^{1 / 2}}
$$

for $\theta^{\prime}$ restricted to $E^{\prime}$ tends to the Gaussian so does $1 /\left|E^{\prime}\right|$ times the distribution function of $Q_{N}\left(\theta^{\prime}\right)$. By the lemma just proved we have $F_{N}(\xi, \eta) /|E|$ $\rightarrow G(\xi, \eta)$ which is the first conclusion of Theorem 2 .

The remaining conclusions of Theorem 2 are proved in the same manner as the first except that the special considerations that were used in $\$ 4$ for each type of series or sum must be employed.

It should be mentioned that Paley developed functional relationships for more general series than just (1.1) and (1.2). The lacunary series on the right then have, however, both coefficients and exponents which are functions of $\theta$. The methods of this paper are not immediately applicable to these series, but we hope to return to the problem on another occasion. 


\section{REFERENCES}

G. H. HaRdy and J. E. LitTlewood

1. Some problems of Diophantine approximation: A remarkable trigonometrial series, Proc. Nat. Acad. Sci. U.S.A. vol. 2 (1916) pp. 583-586.

R. PALEY

1. On Weierstrass's non-differentiable function, Proc. London Math. Soc. vol. 31 (1930) pp. 301-328.

R. SAlem AND A. Zygmund

1. On lacunary trigonometric series, Proc. Nat. Acad. Sci. U.S.A. vol. 33 (1947) pp. 333338.

2. On lacunary trigonometric series II, Proc. Nat. Acad. Sci. U.S.A. vol. 34 (1948) pp. 54-62.

M. WeISS

1. The law of the iterated logarithm for lacunary trigonometric series, Trans. Amer. Math. Soc. vol. 91 (1959) pp. 444-469.

A. ZyGmund

1. Trigonometrical series, Warszawa-Lwów, Monografie Matematyczne, 1935, p. 118.

University of Chicago,

Chicago, Ill. 